\title{
A severe gastroenteritis outbreak of Salmonella enterica serovar Enteritidis PT8, with PFGE profile XbaI.0024 and MLVA profile 2-9-7-3-2 following a christening reception, Greece, 2016
}

\author{
G. MANDILARA ${ }^{1} \dagger$, C. M. VASSALOS ${ }^{2}+$, A. CHRISOSTOMOU ${ }^{3}$, \\ K. KARADIMAS ${ }^{1}$, E. MATHIOUDAKI ${ }^{4}$, T. GEORGAKOPOULOU ${ }^{3}$, \\ S. TSIODRAS ${ }^{5}$ AND K. MELLOU ${ }^{3}$ \\ ${ }^{1}$ National Reference Centre for Salmonella, National School of Public Health, Central Public Health \\ Laboratory/Hellenic Centre for Disease Control and Prevention, Vari, Attica, Greece \\ ${ }^{2}$ European Public Health Microbiology Training (EUPHEM), European Centre for Disease Prevention and \\ Control, Stockholm, Sweden; Greek Health System, Athens, Greece \\ ${ }^{3}$ Hellenic Centre for Disease Control and Prevention, Athens, Greece \\ ${ }^{4}$ Food Unit, Department of Microbiology, Central Public Health Laboratory, Vari, Attica, Greece \\ ${ }^{5}$ Department of Internal Medicine, Atticon University Hospital, National and Kapodistrian University of Athens, \\ Greece
}

Received 5 July 2017; Final revision 18 October 2017; Accepted 3 November 2017; first published online 4 December 2017

\section{SUMMARY}

In June 2016, a Salmonella enterica serovar Enteritidis outbreak $(n=56)$ occurred after a christening reception in Central Greece, mainly affecting previously healthy adults; one related death caused media attention. Patients suffered from profuse diarrhoea, fever and frequent vomiting episodes requiring prolonged hospitalisation and sick leave from work, with a $54 \%$ hospital admission rate. The majority of cases experienced serious illness within $<12 \mathrm{~h}$ of attending the party. We investigated the outbreak to identify the source(s) of infection and contributing factors to the disease severity. From the retrospective cohort study, the cheesy penne pasta was the most likely vehicle of infection (relative risk 7.8; 95\% confidence interval $3 \cdot 6-16 \cdot 8$ ), explaining $79 \%$ of the cases. S. enterica ser. Enteritidis isolates were typed as phage-type PT8, pulsed-field gel electrophoresis type XbaI.0024, multiple locus variable-number tandem repeat analysis-type 2-9-7-3-2. The strain did not share the single-nucleotide polymorphism address of the concurrent European S. enterica ser. Enteritidis PT8 outbreak clusters. Following five consecutive years with no documented $S$. enterica ser. Enteritidis outbreaks in Greece, this outbreak, likely associated with a virulent strain, prompted actions towards the enhancement of the national Salmonella molecular surveillance and control programmes including the intensification of training of food handlers for preventing similar outbreaks in the future. Advanced molecular techniques were useful in distinguishing unrelated outbreak strains.

Key words: Epidemiology, outbreak, Salmonella molecular typing, S. enterica ser. Enteritidis.

\footnotetext{
* Author for correspondence: C. M. Vassalos, European Public Health Microbiology Training (EUPHEM), European Centre for Disease Prevention and Control, Stockholm, Sweden; Greek Health System, Athens, Greece.

(Email: vassalos.constantine@gmail.com)

$\dagger$ Equally contributing first co-authors (names in alphabetical order).
}

\section{INTRODUCTION}

Non-typhoidal salmonellosis usually causes mild, selflimiting gastroenteritis [1]. Symptoms usually appear $12-36 \mathrm{~h}$ after eating and last up to 7 days. The disease can be severe and life-threatening in vulnerable 
populations like children, the elderly and people with underlying comorbidities [2]. Salmonellosis ranks second after campylobacteriosis as the most commonly reported food-borne disease across the European Union (EU) [3, 4]. Despite a significant downward trend in Salmonella infections since 2008, a $15.3 \%$ increase in the overall EU notification rate was noted in 2014 compared with 2013, followed by a further $1.9 \%$ increase in 2015 [3, 4]. Most European cases of non-typhoidal salmonellosis are currently caused by Salmonella enterica serovar Enteritidis [5]. S. enterica ser. Enteritidis is the predominant serovar associated with Salmonella outbreaks [4]. In the EU, in 2015, S. enterica ser. Enteritidis was implicated in $63 \%$ of 953 food-borne outbreaks caused by Salmonella. A Europe-wide large outbreak of $S$. enterica ser. Enteritidis PT8, with multiple locus variable-number tandem repeat analysis (MLVA) profiles 2-9-7-3-2 and 2-9-6-3-2, occurred from May 2016 to February 2017 [5].

In Greece, the mean annual salmonellosis notification rate for the period $2008-2015$ was $4 \cdot 1$ cases per 100000 population, with $S$. enterica ser. Enteritidis being the most common serotype, dropping yearly from $63 \%$ of total isolates in 2008 to $24 \%$ in 2015 [6]. The decreasing trend of $S$. enterica ser. Enteritidis cases was mainly attributed to the Greek National Salmonella Control Programme implemented in poultry in accordance with the EU legislation [7]. At the same period, only small, household, outbreaks of $S$. enterica ser. Enteritidis were identified in the country; the last documented community outbreak was in 2011 (Hellenic Centre for Disease Control and Prevention, data not published).

On 19 June 2016, hospital authorities in Central Greece informed public health authorities of the occurrence of several cases of gastroenteritis following a christening reception at a restaurant in a popular tourist village. A 57-year-old guest with no underlying condition died after being admitted to the hospital with severe gastroenteritis symptoms. Two otherwise healthy guests, aged 26 and 60, were admitted to the intensive care unit. As a consequence, the outbreak captured wide media and public attention. An outbreak control team (OCT) was dispatched to investigate the outbreak and identify the implicated food item, the causative pathogen and factors possibly contributing to disease severity. The OCT also investigated the possible association of the outbreak with the concurrent 2016/17 European outbreak.

\section{METHODS}

\section{Epidemiological investigation}

A retrospective cohort study was performed. Restaurant management provided a list of all food items served. Telephone interviews were conducted by the fellow of the European Public Health Microbiology Training Program of ECDC (EUPHEM) after special training, using a structured questionnaire. Data were obtained on demographics, the symptoms, the need for and the length of hospitalisation, and the time taken off from work. The presence of underlying conditions and the possible contact with ill persons unrelated to the christening party were recorded. To identify potential risk factors for illness, all guests were asked whether they had consumed any of the specified food items. Information on food portions and incubation period was also collected as they were considered to be proxy measures of infectious dose. Foods doses were categorised as 'tasting portion', 'one portion' or 'more than one portion'. For food served on a common platter for four persons, serving size corresponded to about half of a standard portion size. Incubation periods were taken as the time (in hours) from serving the course until the onset of symptoms. After reviewing descriptive data, case was defined as a christening guest who had diarrhoea ( $\geqslant 3$ loose stools in $24 \mathrm{~h}$ ) within $72 \mathrm{~h}$ of the reception.

\section{Statistical analysis}

Categorical variables were compared using the $\chi^{2}$ test. Continuous variables were expressed as median with the corresponding interquartile range, and were compared using the Mann-Whitney $U$ test. $P$-values of $<0.05$ were considered statistically significant. The frequency and intensity of symptoms and association with outcomes were assessed. An epidemic curve was used to depict the length and case distribution of the outbreak. For each consumed food item, attack rate (AR), relative risk (RR) and 95\% confidence interval $(95 \% \mathrm{CI})$ were computed. All analyses were performed using Stata v.12.1 (StataCorp., College Station, Texas, USA).

\section{Laboratory investigation}

Stool samples were screened only for three bacterial pathogens, Salmonella spp., Campylobacter spp. and Shigella spp. The available identified Salmonella isolates were sent to the National Reference Centre for 
Salmonella. Serotyping was performed according to the White-Kaufmann-Le Minor Scheme [8, 9]. Susceptibility testing was carried out using the disk diffusion method. Antimicrobial agents appropriate for monitoring of antibiotic resistance in human Salmonella isolates, according to the relevant EU protocol [10], were tested. The European Committee on Antimicrobial Susceptibility Testing breakpoints were applied [10]. Two outbreak isolates from patients who belonged to different families and were hospitalised at different hospitals, were phage-typed by the Ward-Colindale system [11]. Pulsed-field gel electrophoresis (PFGE) was performed after the digestion of genomic DNA with $\mathrm{XbaI}$ macrorestriction endonuclease, according to standard operating procedure [12]. Similarity and cluster analyses were performed using the Dice coefficient and the unweighted-pair group method with the use of average linkage, using BioNumerics software v.6.0 (Applied Maths, Belgium). PFGE profiles were uploaded to the European Molecular Surveillance System (TESSy-MSS), operated by the European Centre for Disease Prevention and Control, for matching with other characterised PFGE profiles at European level [13]. The isolates were submitted for MLVA analysis, following a laboratory standard operating procedure [14], based on the five-locus MLVA method proposed by Hopkins et al. [15]. Single-nucleotide polymorphism (SNP)-based whole genome sequencing (WGS) analysis was carried out by the PHE Genome Sequencing and Development Unit [16]. A hierarchical, 'SNP address', approach was used to assess the genetic distance between our isolates and those of the concurrent European outbreak [5, 16].

\section{Environmental investigation}

The OCT visited the restaurant where the christening event took place in order to collect information on preparation processes for the foods served. The order and delivery books of the restaurant were reviewed. Environmental swabs were taken from working benches. Food leftovers were no longer available; raw materials were collected for laboratory testing [17]. The OCT also attempted to trace raw materials back to the original suppliers. All the food handlers were interviewed regarding food handling practices and illness 1 week before and after the christening reception. They were also asked whether they had consumed foods served at the reception. Stool samples were obtained after verbal consent from all food handlers.

\section{RESULTS}

\section{Epidemiological investigation}

Of the 133 attendees, 122 (92\%) individuals completed the questionnaire. Participants' median age was $40 \cdot 5$ years (range 3-86); 70 (57\%) participants were females. A total of $56(46 \%)$ respondents met the case definition (median age, 43.5 years (range 3-86); $31(55 \cdot 4 \%)$ females). Of the 56 cases, $49(87 \cdot 5 \%)$ reported high body temperature (median, $39 \cdot 2^{\circ} \mathrm{C}$; range 38.4-41), $37(66 \%)$ abdominal pain and 22 $(39 \cdot 3 \%)$ vomiting, while $30(54 \%)$ were hospitalised. Twenty-eight $(50 \%)$ complained of intense abdominal pain. Eleven $(50 \%)$ of those vomiting had at least four vomiting episodes per day (range 1-15). Half of those requiring hospitalisation stayed at the hospital for 5 days or more (range 1-10 days). Of the $42 / 56$ (75\%) working patients, $21(50 \%)$ reported absenteeism from work, with one out of two being off work for $\geqslant 7$ days (range 1-15 days). Age and sex did not significantly differ between cases and non-cases and were not associated with any of the symptoms and outcomes. Thirty-six cases were previously healthy adults. Eight cases had an underlying condition; cardiovascular disease $(n=4)$; diabetes $(n=3)$; hypothyroidism $(n=1)$. One patient reported a previous upper gastrointestinal tract surgery $(n=1)$. Their clinical presentation and outcome did not differ from those of other cases. None reported achlorhydria or prior use of antacids or acid reducers. Affected individuals had no contact with sick people unrelated to the reception. The shape of the epidemic curve is suggestive of a common point-source outbreak (Graph 1). The median incubation period was $15 \mathrm{~h}$ (range 6-71). Twenty of the $56(35.7 \%)$ patients developed diarrhoeal illness in $<12 \mathrm{~h}$ from serving the course (Table 1). This group of affected guests passed $\geqslant 7$ loose stools in a day, had the highest proportion of fever and vomiting with more than four vomit episodes a day; and had the highest hospitalisation rate lasting $>6$ days. They also had symptoms of long duration ( $\geqslant 8$ days; $n=11,55 \%$ ). Results of the univariate analysis are displayed in Table 2 . A RR of $7 \cdot 8 ; 95 \%$ CI 3.6-16.8 was associated with having eaten cheesy penne pasta at the christening reception. Of the 63 people who ate cheesy penne pasta, 50 fell ill (AR = $79 \cdot 4 \%$ ). As cheesy penne pasta was served on a common platter for four persons, half of a standard portion was allocated for each guest. Most (32/50; 64\%) affected guests, who had eaten cheesy penne pasta, reported that they had eaten less than half-portion. 


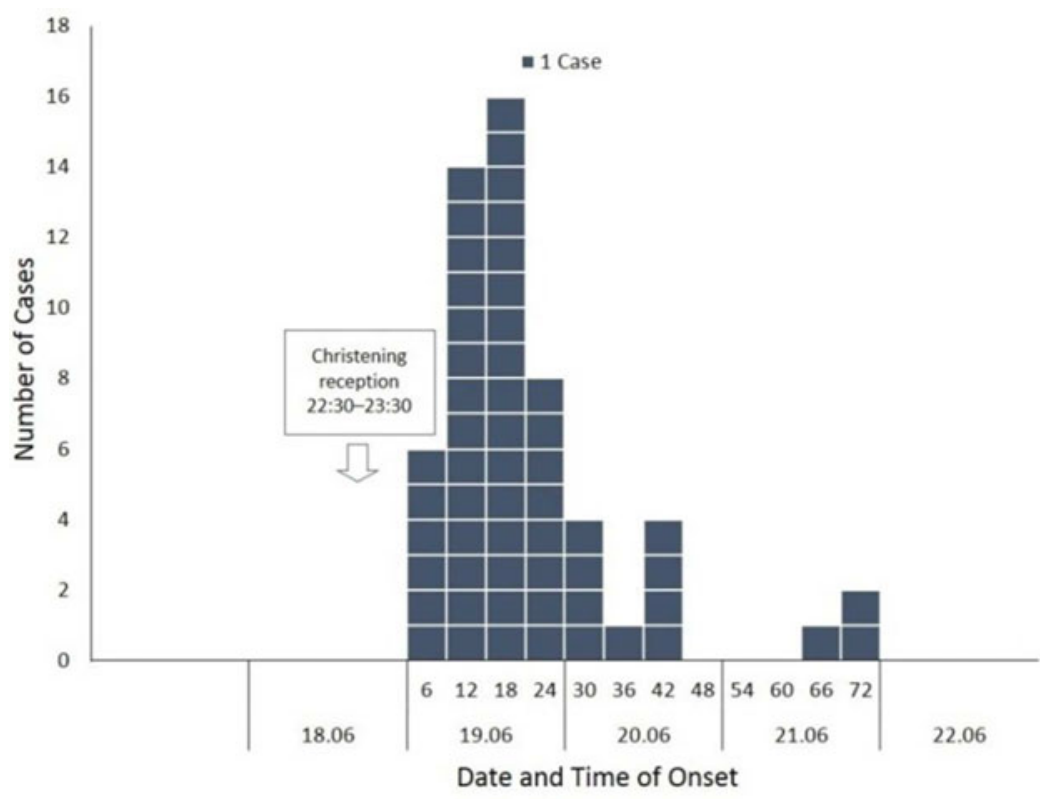

Graph 1. Interviewed guests $(n=56)$ who developed diarrhoea ( $\geqslant 3$ loose stools in 24 hours) within 72 hours following a christening reception in Central Greece, June 2016, by date and six-hour time intervals of onset of illness.

No relationship between symptoms and outcomes with consumed portion of implicated food was shown. One case was reported to have exclusively consumed cheesy penne pasta served at the christening reception.

\section{Laboratory investigation}

Stool cultures from $17 / 56(30 \cdot 4 \%)$ cases were positive for Salmonella spp. Seven isolates were sent for further typing, and were identified as S. enterica ser. Enteritidis. The isolates were found to be fully susceptible to the tested antibiotics. Two isolates were typed as phage-type PT8, with PFGE profile XbaI.0024 and MLVA profile 2-9-7-3-2. The isolates shared the t5-level SNP address 1.2.3.323.323.24095628.\% (with \%-sign substituting any number standing for 0-SNP level) differing from each of the two European outbreak's WGS clusters by 175 (SNP address 1.2.3.175.175.175.\%) and 360 (SNP address 1.2.3.18.359.360.\%).

\section{Other restaurant consumers with $S$. enterica ser. Enteritidis infections}

The food handler that was tested positive for $S$. enterica ser. Enteritidis reported tasting food in the kitchen during the reception (penne pasta) and that he developed symptoms in the following days. It was also found that two persons with laboratory-confirmed $S$. enterica ser. Enteritidis infection had consumed cheesy penne pasta served at the reception despite not attending the event themselves (family members of one of the food handlers). Finally, stool cultures from four clients who fell ill after they had eaten at the restaurant after the reception were positive for $S$. enterica ser. Enteritidis. Data regarding cases other than those among the reception guests are summarised in Table 3.

\section{Environmental investigation}

The restaurant owners had voluntarily closed down their restaurant before the inspection took place. Prior to the arrival of investigators the café kitchen had been cleaned and most perishable goods had been discarded. All environmental and raw materials tested negative for Salmonella. Stool cultures from six out of seven food handlers were also negative for Salmonella. The remaining food handler, who reported diarrhoea after the event, was diagnosed with Salmonella infection. The food handler reported tasting food in the kitchen during the reception. The ingredients of the incriminated dish were penne pasta, yellow hard cheese, semi-hard cheese, cured meat, three packets of crème fraîche, six egg yolks, pepper and salt. The pasta was boiled and cheese was cut at noon. Afterwards, the ingredients were put into the refrigerator. Food was baked at some point in the afternoon. The dish was kept at room 
Table 1. Range of symptoms and outcomes sorted by the length of incubation period among 56 guests falling ill within 72 h after attending a christening reception in Central Greece, June 2016

\begin{tabular}{|c|c|c|c|}
\hline \multirow[b]{2}{*}{ Symptom } & \multicolumn{3}{|l|}{ Incubation period (hours) } \\
\hline & $\begin{array}{l}\leqslant 12 \\
\text { Cases }(n=20 / 56 ; 35 \cdot 7 \%) \\
\text { Number }(\%)\end{array}$ & $\begin{array}{l}>12 \text { to } \leqslant 18 \\
\text { Cases }(n=16 / 56 ; 28 \cdot 6 \%) \\
\text { Number }(\%)\end{array}$ & $\begin{array}{l}>18 \\
\text { Cases }(n=20 / 56 ; 35 \cdot 7 \%) \\
\text { Number }(\%)\end{array}$ \\
\hline Diarrhoea & $20(35 \cdot 7)$ & $16(28 \cdot 6)$ & $20(35 \cdot 7)$ \\
\hline High body temperature $\left(>38 \cdot 3^{\circ} \mathrm{C}\right)$ & $18(32 \cdot 1)$ & $15(26 \cdot 8)$ & $16(28 \cdot 6)$ \\
\hline Malaise/fatigue & $18(32 \cdot 1)$ & $12(21 \cdot 4)$ & $14(25 \cdot 0)$ \\
\hline Abdominal pain & $13(23 \cdot 2)$ & $12(21 \cdot 4)$ & $12(21 \cdot 4)$ \\
\hline Dehydration & $15(26 \cdot 8)$ & $10(17 \cdot 9)$ & $10(17 \cdot 9)$ \\
\hline Vomiting & $16(28 \cdot 6)$ & $5(8 \cdot 9)$ & $1(1 \cdot 8)$ \\
\hline Dizziness/loss of consciousness & $7(12 \cdot 5)$ & $1(1 \cdot 8)$ & $4(7 \cdot 1)$ \\
\hline Outcome & Number $(\%)$ & Number $(\%)$ & Number $(\%)$ \\
\hline Hospitalisation & $17(30 \cdot 4)$ & $6(10 \cdot 7)$ & $7(12 \cdot 5)$ \\
\hline Taking time off from work ${ }^{\mathrm{a}}$ & $8(19 \cdot 0)$ & $10(23 \cdot 8)$ & $3(7 \cdot 2)$ \\
\hline
\end{tabular}

${ }^{\mathrm{a}}$ The results refer to 42 cases of working age.

Table 2. Food-specific attack rate and relative risk of developing diarrhoea by food item among 122 persons who attended a christening reception in Central Greece, June 2016

\begin{tabular}{|c|c|c|c|c|c|c|c|c|}
\hline \multirow[b]{2}{*}{ Consumption of specified food item } & \multicolumn{3}{|c|}{ Exposed } & \multicolumn{3}{|c|}{ Unexposed } & \multirow[b]{2}{*}{$\mathrm{RR}$} & \multirow[b]{2}{*}{$95 \% \mathrm{CI}$} \\
\hline & Cases & Total & AR \% & Cases & Total & AR $\%$ & & \\
\hline Savoury pie with cheese & 47 & 95 & $49 \cdot 5$ & 7 & 24 & $29 \cdot 2$ & $1 \cdot 69$ & $0 \cdot 88-3 \cdot 27$ \\
\hline Savoury pie with spinach & 34 & 68 & $50 \cdot 0$ & 21 & 53 & $39 \cdot 6$ & $1 \cdot 26$ & $0 \cdot 84-1 \cdot 90$ \\
\hline Aubergine with minced meat and béchamel & 46 & 91 & $50 \cdot 5$ & 9 & 30 & $30 \cdot 0$ & 1.68 & $0 \cdot 94-3 \cdot 02$ \\
\hline Vineyard leaves filled with rice & 35 & 76 & $46 \cdot 1$ & 20 & 45 & $44 \cdot 4$ & $1 \cdot 04$ & $0 \cdot 69-1 \cdot 56$ \\
\hline Burger & 46 & 93 & $49 \cdot 5$ & 9 & 28 & $32 \cdot 1$ & $1 \cdot 54$ & $0 \cdot 86-2 \cdot 74$ \\
\hline Stew meat & 24 & 45 & $53 \cdot 3$ & 30 & 75 & $40 \cdot 0$ & $1 \cdot 33$ & $0 \cdot 90-1 \cdot 97$ \\
\hline Cheesy penne pasta & 50 & 63 & $79 \cdot 4$ & 6 & 59 & $10 \cdot 2$ & $7 \cdot 80$ & $3 \cdot 62-16 \cdot 8$ \\
\hline Greek salad & 17 & 37 & $45 \cdot 9$ & 38 & 84 & $45 \cdot 2$ & $1 \cdot 02$ & $0 \cdot 67-1 \cdot 55$ \\
\hline Green salad & 17 & 32 & $53 \cdot 1$ & 38 & 89 & $42 \cdot 7$ & $1 \cdot 24$ & $0 \cdot 83-1 \cdot 87$ \\
\hline Feta cheese & 20 & 35 & $57 \cdot 1$ & 34 & 84 & $40 \cdot 5$ & $1 \cdot 41$ & $0 \cdot 96-2 \cdot 08$ \\
\hline Dip with cheese and pepper & 22 & 40 & $55 \cdot 0$ & 33 & 81 & $40 \cdot 7$ & $1 \cdot 35$ & $0 \cdot 92-1 \cdot 98$ \\
\hline Oven-roasted potatoes & 17 & 30 & $56 \cdot 7$ & 35 & 88 & $39 \cdot 8$ & $1 \cdot 42$ & $0 \cdot 95-2 \cdot 14$ \\
\hline Biscuit cake & 24 & 60 & $40 \cdot 0$ & 32 & 60 & $53 \cdot 3$ & $0 \cdot 75$ & $0 \cdot 51-1 \cdot 11$ \\
\hline Cheesecake & 24 & 44 & $54 \cdot 5$ & 32 & 73 & $43 \cdot 8$ & $0 \cdot 95$ & $0 \cdot 64-1 \cdot 42$ \\
\hline Chocolate pastry & 24 & 43 & $55 \cdot 8$ & 35 & 81 & $43 \cdot 2$ & $1 \cdot 24$ & $0 \cdot 86-1 \cdot 81$ \\
\hline Homemade cake (lemon pie) & 5 & 13 & $38 \cdot 5$ & 46 & 98 & $46 \cdot 9$ & $0 \cdot 82$ & $0 \cdot 40-1 \cdot 68$ \\
\hline Chocolate fondue fountain & 4 & 11 & $36 \cdot 4$ & 49 & 105 & $46 \cdot 7$ & $0 \cdot 78$ & $0 \cdot 35-1 \cdot 75$ \\
\hline
\end{tabular}

Food specific attack rates (AR), relative risks (RR) and 95\% confidence intervals $(95 \% \mathrm{CI})$ were calculated.

temperature for more than $2 \mathrm{~h}$ before being served after the completion of the first course. It has to be mentioned that the outbreak occurred during a heat wave in Greece. The temperature at the day of the reception ranged between $25 \cdot 7$ and $39 \cdot 5{ }^{\circ} \mathrm{C}$. Inspectors recorded the lack of a Hazard Analysis and Critical Control Point system and of standard procedures inside the kitchen for avoiding cross-contamination.
Raw and cooked foods were not adequately separated. The oven's heating performance was not tested; no registry data were kept on refrigerator temperature. The origin of the eggs used to prepare the cheesy penne pasta was also investigated. An inspection of the implicated hatchery and packing station was initiated. Samples taken were negative for S. enterica spp. 


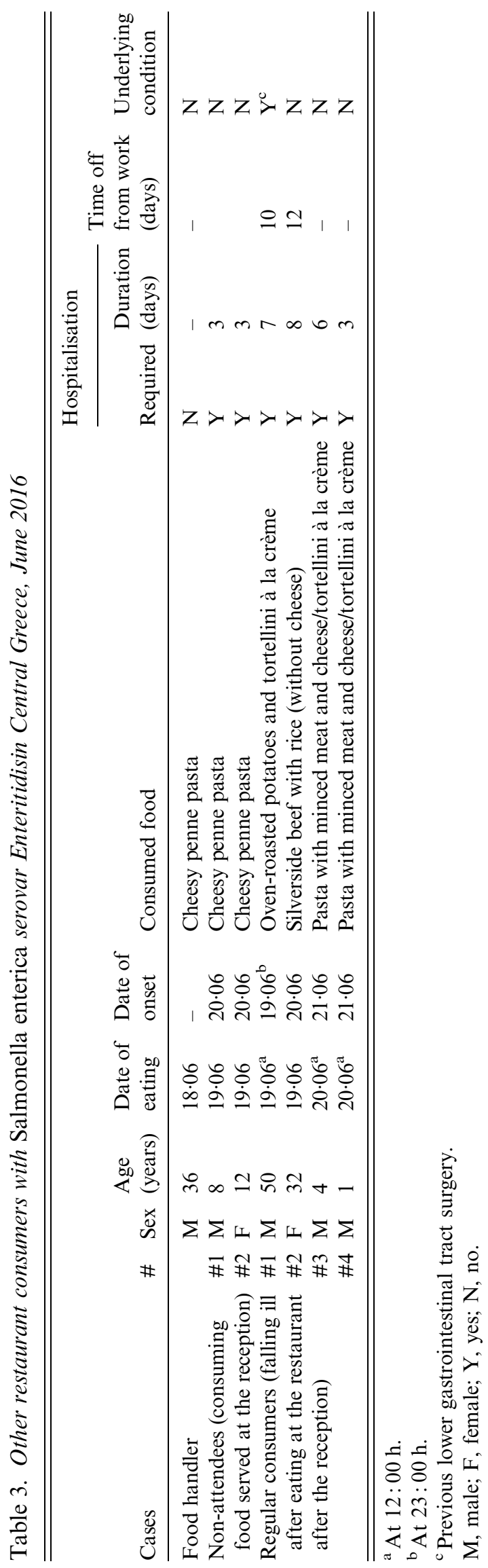

\section{Measures taken}

The local public health authorities suspended the license of the restaurant for 4 months. The suspension was lifted after a thorough review of the restaurant's food safety management system and the implementation of improvements to enhance processes associated with reduction in the potential for food contamination (focus at monitoring and recording of processes and temperatures, heat treatment and preservation of food). Furthermore, all employees were re-trained on the basic concepts of Hazard Analysis and Critical Control Point (HACCP) and their compliance was further assessed by the local public health authority. The enhancement of the national Salmonella molecular surveillance was also decided; MLVA typing will be introduced in the routine work of the National Reference for salmonellosis.

\section{DISCUSSION}

A serious $S$. enterica ser. Enteritidis PT8, MLVA profile 2-9-7-3-2 outbreak occurred in Greece in 2016, despite a recorded decreasing trend of cases in the previous years and the absence of recorded large outbreaks due to this serotype in the country since 2011. Remarkable morbidity was associated with this outbreak, even though non-typhoidal salmonellosis is generally mild. High rates of severe symptoms and hospital admission were reported. Patients suffered from severe gastrointestinal symptoms such as profuse diarrhoea, fever and frequent vomiting episodes demanding prolonged hospitalisation and sick leave from work. One death was associated with this outbreak. Another interesting feature was that the majority of affected guests experienced serious gastrointestinal illness within $<12 \mathrm{~h}$ of attending the reception. There has been an inconclusive debate about the infectious dose and illness severity of nontyphoidal Salmonella [18-20]. In this outbreak, the small amount of consumed cheesy penne pasta did not allow us to assess the dose-response relationship. It is possible that bacteria may have survived inadequate heat processing and high-fat cheesy penne pasta may have protected Salmonella cells - encapsulated in fat - against stomach acidity [21]. Another possible explanation is that leaving this perishable food out of the refrigerator on a warm summer evening allowed bacterial multiplication in the likely vehicle of infection [22]. The short incubation period may be attributable to very high infectious dose and/ 
or co-infection with toxin-producing bacteria (e.g. Staphylococcus aureus), as described before [23]; their isolation though was not feasible in Greek local hospitals at that point of time. The severity of Salmonella-associated disease may additionally depend on host factors as well as the specific strain of Salmonella [24]. Regarding host status in the current outbreak, more than $60 \%$ of the cases were otherwise healthy adults. Over the last two decades, only a limited number of serious $S$. enterica ser. Enteritidis outbreaks affecting mainly non-high-risk adults have been published [19, 20, 25-28]. The fact that severe $S$. enterica ser. Enteritidis outbreaks are infrequent is perhaps in line with the serovar's weak human invasiveness in high-income countries [29]. Finally, the outbreak-associated isolates were found to belong to phage-type PT8.

Interestingly, despite lacking correlation between clonal lineage and virulence within the serovar, PT8 has been suggested to be one of the high virulent $S$. enterica ser. Enteritidis phage types [30, 31]. Our finding that the isolates of the present outbreak were identified as PT8 and produced the PFGE profile XbaI.0024 according to TESSy-MSS (corresponding to PFGE profile SENTXB.0002 according to the former PulseNet Europe network for food-borne infections in Europe) is consistent with studies showing a strong association between PT8 and SENTXB.0002 [32]. Phage-type PT8 has been one of the most frequently observed phage types in Eastern Europe since the 1990s [26, 33-37].

In 2011-2015, PT8 was the predominant phage type in human cases in 13 European countries [5]; no such data were available for Greek cases. In 2016, a gastroenteritis outbreak occurred amid a $S$. enterica ser. Enteritidis PT8 outbreak affecting different countries in Europe [5]. The MLVA profile of our PT8 isolates was indistinguishable from the MLVA profile 2-9-7-3-2 of the multi-country outbreak [5]. SNP-based WGS analysis, however, revealed that our outbreak did not belong to either of the two WGS clusters associated with the 2016/17 European outbreak despite their temporal relatedness.

Information on the factors that contributed to the occurrence of the outbreak involved the high ambient temperature at the time of the outbreak and the lack of standard procedures inside the kitchen for avoiding cross-contamination. The fact that four people fell ill after they had eaten at the restaurant the days following the reception is supportive of the hypothesis that probably cross-contamination occurred inside the kitchen during the preparation for the reception. Even though cross-contamination after cooking is the most probable cause of the outbreak, the exact point that cross-contamination occurred remained unclear. The literature shows that this is a common limitation of the investigation of outbreaks at restaurant premises [38]. For this reason, environmental investigation of similar outbreaks should be more detailed, and inspection should be scheduled as soon as possible after the establishment being linked with an outbreak, ideally within a day. It is also recommended that the authorities conduct multiple establishment visits if needed to complete the environmental assessment and monitor poor handling practices. In this case due to the media attention, the restaurant owners had voluntarily closed down their restaurant before the inspection took place and investigators were restricted in their ability to hypothesise and test potential means of cross-contamination during the preparation of the incriminated meal. That most Greek local hospitals lacked the capacity to screen stools for other food-borne pathogens with very short incubation periods (at least shorter than that of Salmonella), such as $S$. aureus, constitutes one more limitation, since co-infection cannot be excluded. Finally, the current study is limited by the fact that no leftovers were available for microbiological testing and identification of the food vehicle.

\section{CONCLUSION}

In the aftermath of the herein described recent $S$. enterica ser. Enteritidis outbreak associated with high morbidity, after five consecutive years of no outbreak documentation, the occurrence of $S$. enterica ser. Enteritidis outbreaks remains a public health threat in Greece. Thus, despite the progress in Salmonella control, there can be no complacency; it is vitally critical to maintain and strengthen the continued implementation of the National Salmonella Control Programmes and to intensify efforts to (re) educate food handlers in the basic principles of food management and hygiene to prevent such outbreaks from occurring. A more detailed environmental investigation is needed in case of similar outbreaks so that the factors led to cross-contamination inside the kitchen be identified and the appropriate correction measures be taken. Advanced molecular data need to be incorporated into the current public health surveillance systems in Greece to help us distinguish unrelated outbreak strains and identify emerging clones. 


\section{ACKNOWLEDGEMENTS}

The authors are thankful to the christening guests and the restaurant staff for their kindness in answering their questions. The authors would like to acknowledge Silvia Herrera-León, in Sección de Enterobacterias, Servicio de Bacteriología, Centro Nacional de Microbiología, Instituto de Salud Carlos III, Madrid, Spain, and Aftab Jasir, European Centre for Disease Prevention and Control, Stockholm, Sweden, for their reviewing the manuscript. The authors also thank Gitte Sørensen, Research Group for Genomic Epidemiology, National Food Institute, Technical University of Denmark, Lyngby, Denmark and Tim Dallman at Public Health England, UK for phage typing and whole genome sequencing the outbreak isolates, respectively; Saara Kotila, Scientific Officer Molecular Surveillance Epidemiological Methods, SRS, European Centre for Disease Prevention and Control, Stockholm, Sweden for all her support. The authors are also thankful to Ioannis G. Koutelekos, Faculty of Nursing, Technological Institute of Education, Athens, Greece for his entering data in worksheet cells and preparing tables in the manuscript. The authors acknowledge Alkiviadis Vatopoulos, National School of Public Health, Athens, and Kyriaki Tryfinopoulou, Central Public Health Laboratory, Vari, Attica, Greece, for their remarks. The authors also thank the Directorate of Health and Social Care, and the Hospital Authorities, Boeotia Regional Unit, Central Greece Region; and the Hellenic Food Authority for supporting OCT investigation.

\section{DECLARATION OF INTEREST}

None.

\section{REFERENCES}

1. Heymann DL. Control of Communicable Diseases Manual, 19th edn. Washington, DC: APHA Press, 2008.

2. Hunter JC, Francois Watkins LK. Salmonellioss (nontyphoidal). In: Centers for Disease Control and Prevention. Yellow Book 2018. Chapter 3. Centers for Disease Control and Prevention, Atlanta, Georgia. Available from. https://wwwnc.cdc.gov/travel/yellowbook/2018/ infectious-diseases-related-to-travel/salmonellosisnontyphoidal

3. European Food Safety Authority/European Centre for Disease Prevention and Control. The European Union summary report on trends and sources of zoonoses, zoonotic agents and food-borne outbreaks in 2014. EFSA Journal 2015; 13: 4329.

4. European Food Safety Authority/European Centre for Disease Prevention and Control. The European Union summary report on trends and sources of zoonoses, zoonotic agents and food-borne outbreaks in 2015. EFSA Journal 2016; 14: 4634.

5. European Food Safety Authority/European Centre for Disease Prevention and Control. Multi-country outbreak of Salmonella Enteritidis phage type 8, MLVA type 2-9-7-3-2 and 2-9-6-3-2 infections [Technical report]. Published: 7 March 2017.

6. Hellenic Centre for Disease Control and Prevention. Annual salmonellosis report, 2015. Available from: http://www.keelpno.gr/Portals/0/Files/English\%20files/ Reports\%20(annual-periodical)/Epidemiological\%20data $\%$ 20for\%20Salmonellosis_2004-2015.pdf.

7. Ministry of Rural Development and Food. Implementation of the National Control Programmes for Salmonella in chicken and turkey flocks in the years 2009-2014. [Technical report]. Published: 23 December 2015.

8. ISO/TR 6579-3:2014 (E). Microbiology of food and animal feed-Horizontal method for the detection, enumeration and serotyping of Salonella-Part3 Guidelines for serotyping of Salmonela. International Organization for Standardization, Geneva, Switzerland. Published: 31 July 2014.

9. Grimont PAD, Weill F-X. Antigenic Formulae of the Salmonella Serovars, 9th edn. Paris: World Health Organization Collaborating Centre for Reference and Research on Salmonella; 2007.

10. European Centre for Disease Prevention and Control. $E U$ Protocol for Harmonized Monitoring of Antimicrobial Resistance in Human Salmonella and Campylobacter Isolates - June 2016. Stockholm: ECDC; 2016.

11. Ward LR, de Sa JD, Rowe B. A phage-typing scheme for Salmonella Enteritidis. Epidemiology and Infection 1987; 99: 291-294.

12. Centers for Disease Control and Prevention (CDC). Standard Operating Procedure for PulseNet PFGE of Escherichia coli O157:H7, Escherichia coli non-O157 (STEC), Salmonella serotypes, Shigella sonnei and Shigella flexneri. Atlanta: CDC; Apr 2013. Available from: http://www.cdc.gov/pulsenet/pdf/ecoli-shigellasalmonella-pfge-protocol-508c.pdf.

13. European Centre for Disease Prevention and Control. The European Surveillance System (TESSy) [Database]. Stockholm: ECDC; 2016.

14. European Centre for Disease Prevention and Control. Laboratory Standard Operating Procedure for Multiple-locus Variable-Number Tandem Repeat Analysis of Salmonella enterica SEROTYPE ENTERITIDIS. [Technical document]. Stockholm: ECDC; 2016.

15. Hopkins KL, et al. Standardisation of multilocus variable-number tandem-repeat analysis (MLVA) for subtyping of Salmonella enterica serovar Enteritidis. Eurosurveillance 2011; 16: pii=19942.

16. Inns T, et al. Prospective use of whole genome sequencing (WGS) detected a multi-country outbreak of 
Salmonella Enteritidis. Epidemiology and Infection 2017; 145: 289-298.

17. ISO 6579:2002. Microbiology of food and animal feedhorizontal method for the detection of Salmonella spp. International Organization for Standardization, Geneva, Switzerland. Published: 15 July 2002.

18. Glynn JR, Brandley DJ. The relationship between infecting dose and severity of disease in reported outbreaks of Salmonella infections. Epidemiology and Infection 1992; 109: 371-388.

19. Rejnmark L, et al. Impact of infecting dose on severity of disease in an outbreak of food-borne Salmonella Enteritidis. Scandinavian Journal of Infectious Diseases 1997; 29: 37-40.

20. Mintz ED, et al. Dose-response effects in an outbreak of Salmonella Enteritidis. Epidemiology and Infection 1994; 112: $13-23$.

21. Álvarez-Ordóñez A, et al. Salmonella spp. survival strategies within the host gastrointestinal tract. Microbiology 2011; 157: 3268-3281.

22. D'Argenio P, Romano A, Autorino F. An outbreak of Salmonella Enteritidis infection associated with iced cake. Eurosurveillance 1999; 4: 24-26.

23. Huang AD, et al. Metagenomics of two severe foodborne outbreaks provides diagnostic signatures and signs of coinfection not attainable by traditional methods. Applied and Environmental Microbiology 2017; 83: e02577-16.

24. World Health Organization. Salmonella (non typhoidal). Fact sheet. Updated December 2016. Available from: http://www.who.int/mediacentre/factsheets/fs139/en/.

25. Cerný Z, Bazoutová M. An epidemic of salmonellosis in the First League Hockey Team. [In Czech]. Epidemiologie, Mikrobiologie, Imunologie: Casopis Spolecnosti pro Epidemiologii a Mikrobiologii Ceske Lekarske Spolecnosti JE Purkyne 1998; 47: 166-168.

26. Garcia-Huidobro D, et al. Clinical and epidemiological description of severe outbreak of foodborne infection by Salmonella Enteritidis. [In Spanish]. Revista Chilena de Infectologia: Organo Oficial de la Sociedad Chilena de Infectologia 2012; 29: 132-137.

27. Giraudon I et al. Large outbreak of Salmonella phage type 1 infection with high infection rate and severe illness associated with fast food premises. Public Health 2012: 123: 444-447.
28. Mertens E, et al. Severe infections caused by Salmonella Enteritidis PT8/7 linked to a private barbecue. Epidemiology and Infection 2013; 141: 277-283.

29. Feasay NA, et al. Distinct Salmonella Enteritidis lineages associated with enterocolitis in high-income settings and invasive disease in low-income settings. Nature Genetics 2016; 48: 1211-1217.

30. Pang JC, et al. The presence of major world-wide clones for phage type 4 and 8 Salmonella enterica serovar Enteritidis and the evaluation of their virulence levels by invasiveness assays in vitro and in vivo. FEMS Microbiology Letters 2006; 263: 148-154.

31. Olsen JE, Tiainen T, Brown DJ. Levels of virulence are not determined by genomic lineage of Salmonella enterica serotype Enteritidis strains. Epidemiology and Infection 1999; 123: 423-430.

32. Peters TM, et al. Relationship of pulsed-field profiles with key phage types of Salmonella enterica serotype Enteritidis in Europe: results of an international multicentre study. Epidemiology and Infection 2007; 135: 1274-1284.

33. Cieslik A, et al. Phage types and plasmid profiles of plasmid DNA strains of Salmonella enterica subsp. enterica ser. Enteritidis (S. Enteritidis) isolated from food poisoning outbreaks in 2001. [In Polish]. Medycyna Oswiadczalna Mikrobiologia 2002; 54: 325-334.

34. Majtanova L. Occurrence of phage types of nontyphoid Salmonella serovar in the Slovak Republic 2000-2003 [In Slovak]. Epidemiologie Mikrobiologie Imunologie 2004; 53: 70-73.

35. Majtanova L. Occurrence of Salmonella enterica serotype Enteritidis phage types in the Slovak Republic. European Journal Epidemiology 1997; 13: 243-245.

36. Nygård $\mathbf{K}$, et al. Emergence of new Salmonella Enteritidis phage types in Europe? Surveillance of infections in returning travellers. BMC Medicine 2004; 2: 32 .

37. Karpiskova R, Mikulaskova M. Salmonella phage types distribution in the Czech Republic in 1991-1994. Central European Journal Public Health 1995; 3: 161-162.

38. Brown LG, et al. Outbreak characteristics associated with identification of contributing factors to foodborne illness outbreaks. Epidemiology and Infection 2017; 145(11): 2254-2262. 